EPJ Web of Conferences 71, 00097 (2014)

DOI: 10.1051/epjconf/20147100097

(C) Owned by the authors, published by EDP Sciences, 2014

\title{
Fate of inflation and the natural reduction of vacuum energy
}

\author{
Akika Nakamichi ${ }^{1, a}$ and Masahiro Morikawa ${ }^{2, b}$ \\ ${ }^{1}$ Koyama Observatory, Kyoto-Sangyo University, Kyoto 603-8555 JAPAN \\ ${ }^{2}$ Department of Physics, Ochanomizu University, Tokyo 112-0012 JAPAN
}

\begin{abstract}
In the standard cosmology, an artificial fine tuning of the potential is inevitable for vanishing cosmological constant, though slow-rolling uniform scalar field easily causes cosmic inflation. We focus on the general fact that any potential with negative region can temporally halt the cosmic expansion at the end of inflation, where the field tends to diverge. This violent evolution naturally causes particle production and strong instability of the uniform configuration of the fields. Decaying of this uniform scalar field would leave vanishing cosmological constant as well as locally collapsed objects. The universe then continues to evolve into the standard Freedman model. We study the detail of the instability, based on the linear analysis, and the subsequent fate of the scalar field, based on the non-linear numerical analysis. The collapsed scalar field would easily exceed the Kaup limiting mass and forms primordial black holes, which may play an important role in galaxy formation in later stages of cosmic expansion. We systematically describe the above scenario by identifying the scalar field as the boson field condensation (BEC) and the inflation as the process of phase transition of them.
\end{abstract}

\section{1 introduction}

The inflation in the early stage of the Universe is believed to have created the large, causal, and diverse Universe we observe at present from the microscopic quantum origin. The concept of the inflationary scenario of cosmology will be true but the basic problems are still open. They are the (a) initiation and the (b) termination of the inflation, and the (c) problem of the cosmological term (zero-point fine tuning problem) [1].

On the other hand, we have other fundamental problems in the present Universe. They are the (d) origin of the dark energy (DE) and the (e) identification of dark matter (DM). Considering the possibility that these problems (d), (e) have a common origin, we have once proposed a model based on the Bose-Einstein condensation (BEC) of a light boson field [2]. In this model, DM is identified as boson gas and the condensation of it yields DE. This non-adiabatic condensation process is the heart of this model.

In this paper, we would like to extend this BEC model to the early Universe, concentrating on the problems (a), (b), and (c). BEC of the boson gas, which may or may not be related to that in the present Universe, yields the inflaton classical field which causes the inflation. The problem (c) is notorious

\footnotetext{
ae-mail: nakamichi@cc.kyoto-su.ac.jp

be-mail: hiro@phys.ocha.ac.jp
} 
for many years when considered within the unitary evolution[1]. However, the non-adiabatic process of BEC may give some light on this problem.

BEC of the boson gas simply describes the initiation of the inflation (a), and any potential which has some portion of the negative region can always cause a common behavior called stagflation[3], in which the cosmic expansion temporarily ceases while the inflaton still develops. This solves the problem (b), but the strong instability at this stage remains. We would like to use this instability affirmatively to solve the problem (c); the strong instability yields the collapse of the uniform BEC to the localized structures, part of which may be the primordial black holes, thus avoiding the original instability of the uniform space-time.

In the next section, a basic set of equations which describes BEC and inflaton is introduced. The section 3 describes the generality of the stagflation phase for various potentials in this model. Then in section 4, we analyze the instability of the stagflation against the decay of the homogeneity of BEC and the collapse of it to the localized states including the possibility of the primordial black holes. The final section 5 is devoted to the summary of the present work.

\section{Initiation of the inflation - BEC cosmology}

We consider the following set of equations for the scale factor $a(t)$, the boson gas DM energy density $\rho_{\text {gas }}$, and the energy density of the boson condensation as $\mathrm{DE}(=\mathrm{BEC}) \rho_{\phi}$,

$$
\begin{aligned}
H^{2} & \equiv\left(\frac{\dot{a}}{a}\right)^{2}=\frac{8 \pi G}{3 c^{2}}\left(\rho_{\text {gas }}+\rho_{\phi}\right), \\
\dot{\rho}_{\text {gas }} & =-4 H \rho_{\text {gas }}-\Gamma \rho_{\text {gas }}+\gamma \dot{\phi}^{2}, \\
\dot{\rho}_{\phi} & =-6 H\left(\rho_{\phi}-V\right)+\Gamma \rho_{\text {gas }}-\gamma \dot{\phi}^{2},
\end{aligned}
$$

where the interaction term $V$ and $\rho_{\phi}$ are given by

$$
\begin{aligned}
V & =\frac{m^{2}}{2} \phi^{2}+\frac{\lambda}{4} \phi^{4}+V_{0}, \\
\rho_{\phi} & =\frac{\dot{\phi}^{2}}{2}+V,
\end{aligned}
$$

with $\lambda(\equiv-\kappa)<0$ and $V_{0} \gg m^{2}>0$. The phenomenological parameters $\Gamma, \gamma$ represent the BEC condensation speed and the particle creation rate, respectively. The potential $V$ is assumed to have some negative region, which yields the stagflation.

The Universe is naturally assumed to have started with large amount of $\rho_{\text {gas }}$, and then this field have yielded BEC $\phi$. This is the initiation of the inflation, i.e. the slow rolling of the classical scalar field. The approximate analytic solution is given, from Eqs.(1- 5), as

$$
\phi_{\text {inf }}(t) \propto\left(\frac{3 H}{2 \kappa}\right)^{1 / 2}\left(t_{0}-t\right)^{-1 / 2},
$$

which describes the early stage of the inflation evolution.

\section{Termination of the inflation - the stagflation}

The inflation never continues forever. The condensation field $\phi$ eventually evolves into the negative potential region of $V$. Then the whole variables experience the temporal phase 

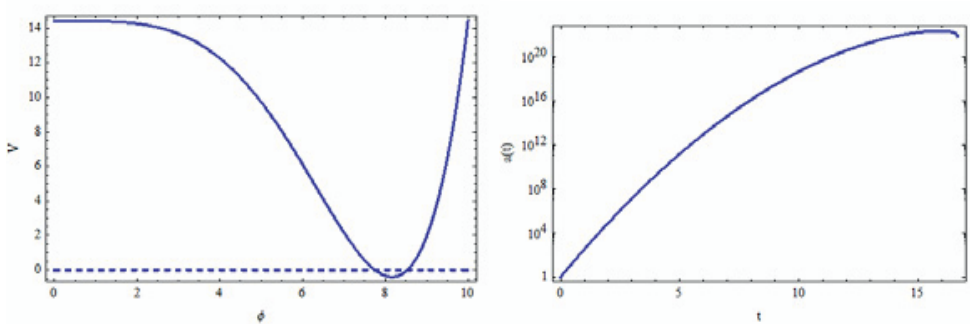

Figure 1. (left) General potential $V$ which has a negative region. Time evolution in this potential, according to Eqs.(1-5) always yields stagflation. (right) The stagflation yielded by the left potential. After almost the exponential expansion of inflation, the scale factor $a(t)$ ceases for a while befor it implode, if we assume the uniform space.

$$
\rho_{\phi}=0, \quad \dot{\rho}_{\phi}=0, \quad H=0,
$$

quite generally provided the potential has some portion of negative region[6]. On the other hand the field $\phi$ continues to evolve, thus this phase is called stagflation. The occurence of the phase Eq.(7) is general because the approximate equation

$$
\dot{H}=-\frac{4 \pi G}{c^{2}} \dot{\phi}^{2}
$$

holds, from Eqs.(1-5), and $\dot{\phi}$ is always finite. This is a very characteristic feature of our model compared with the standard inflationary models which assumes the lowest potential value being naught.

After some excursion in the stagflation phase, the Universe turns into the very strong instability of implosion. The approximate behavior during this implosion is given by

$$
\phi_{\text {imp }}(t) \propto \text { const. }-\frac{\sqrt{2}}{3 \kappa} \log \left(t_{*}-t\right) .
$$

This implosion instability takes place only if considered in the spatially homogenous models. However actually before this implosion phase actually takes place, the stagflation phase becomes unstable against the formation of inhomogeneous structures, which helps to avoid the implosion of the whole Universe. This is the subject we now study in the next section.

\section{Collapse of BEC and formation of localized structures - Instability of stagflation}

We analyze the linearized version of the basic set of equations (1-5) [4]. We decompose the variables as

$$
\begin{aligned}
\phi(t, \vec{r}) & =\phi_{0}(t)+\phi_{1} \exp (\Omega t+i \vec{k} \vec{r}), \\
\Phi(t, \vec{r}) & =\Phi_{0}(t)+\Phi_{1} \exp (\Omega t+i \vec{k} \vec{r}), \\
\rho_{\text {gas }}(t, \vec{r}) & =\rho_{\text {gas } 0}(t)+\rho_{1} \exp (\Omega t+i \vec{k} \vec{r}),
\end{aligned}
$$


where the index naught indicates the background solution which is assumed to be known. Then we obtain the following perturbation equation for four real variables $\operatorname{Re} \phi_{1}, \operatorname{Im} \phi_{1}, \Phi, \rho_{\text {gas }}$,

$$
\begin{aligned}
\left\{\Omega^{2}+3 H \Omega+\frac{k^{2}}{a^{2}}+m^{2}+3 \lambda\left|\phi_{0}\right|^{2}\right\} \phi_{1}^{\mathrm{Re}}+2 \phi_{0}\left(m^{2}+\lambda\left|\phi_{0}\right|^{2}\right) \Phi_{1} & =0 \\
\left\{\Omega^{2}+3 H \Omega+\frac{k^{2}}{a^{2}}+m^{2}+\lambda\left|\phi_{0}\right|^{2}\right\} \phi_{1}^{\mathrm{Im}} & =0 \\
-4 \pi G a^{2}\left\{2 \dot{\phi}_{0} \Omega+2 m^{2} \phi_{0}+2 \lambda\left|\phi_{0}\right|^{3}\right\} \phi_{1}^{\mathrm{Re}}+\left\{\Omega^{2}+\frac{k^{2}}{a^{2}}+3 H \Omega\right\} \Phi_{1} & =0 \\
2 \gamma \dot{\phi}_{0} \Omega \phi_{1}^{\mathrm{Re}}+\{\Omega+4 H\} \rho_{1} & =0
\end{aligned}
$$

The existence of the non-trivial solution demands the following condition for the parameter $\Omega$,

$$
\begin{array}{r}
0=(4 H+\Omega)\left(\frac{k^{2}}{a^{2}}+3 H \Omega-\kappa \phi^{2}+m^{2}+\Omega^{2}\right) \times \\
\left(16 \pi a^{2} G \phi\left(m^{2}-\kappa \phi^{2}\right)\left(-\kappa \phi^{3}+m^{2} \phi+\Omega \dot{\phi}\right)+\left(\frac{k^{2}}{a^{2}}+\Omega(3 H+\Omega)\right)\left(\frac{k^{2}}{a^{2}}+3 H \Omega-3 \kappa \phi^{2}+m^{2}+\Omega^{2}\right)\right)
\end{array}
$$

which yields the instability time scale $1 / \Omega$ in terms of the spatial scale of instability $a / k$.

It is important to specify the spatial scale which actually collapses to form localized structures. Since the collapse speed never exceeds that of light $(c=1)$, the actual collapse takes place when the spatial scale is smaller than the instability time scale times the speed of light. This condition yields $\Omega<k / a$. Furthermore, among those modes, the smallest time scale or the largest $\Omega$ dominates the collapse. Therefore we obtain the condition for the special scale at which the actual collapse takes place:

$$
\Omega\left(k_{*}\right)=k_{*} / a .
$$

A simplest instability is triggered by the attractive interaction $(\kappa)$ of the fields, as is calculated from Eq.(17),

$$
k_{*}=\frac{\sqrt{m_{e}^{2}-2 m^{2}}}{\sqrt{6}} \approx \sqrt{\frac{\kappa}{2}} \phi
$$

where $m_{e}^{2} \equiv-m^{2}+3 \kappa \phi^{2}$ is the negative of the effective mass. On the other hand the standard gravity also triggers instability yielding the scale (inverse of the Jeans length)

$$
k_{*}=4 \sqrt{2} \pi G m \phi^{2} .
$$

More general case is the complex mixture of these two kinds of instability,

$$
k_{*}=\frac{1}{2} \sqrt{\sqrt{-\frac{64 \pi G m^{4}\left(m_{e}^{2}+m^{2}\right)}{3 \kappa}-2 m^{2}\left(m_{e}^{2}+m^{2}\right)-64 \pi G \kappa^{2} \phi^{6}+128 \pi G \kappa m^{2} \phi^{4}+9 \kappa^{2} \phi^{4}+m^{4}}+m_{e}^{2}} .
$$

Both of them Eqs.(19,20) can yield sufficiently small scale of collapse well inside the Horizon scale when the field $\phi$ well develops although the detail will need much elaborate analysis.

Furthermore, since the field $\phi$ is evolving during the stagflation phase, we need to choose the background fields $\phi_{0}(t), \Phi_{0}(t), \rho_{\text {gas } 0}(t)$ which are not static. We solve the background fields near the stagflation point,

$$
\phi_{0}(t)=\frac{1}{-c_{1}-\sqrt{\kappa} t}
$$




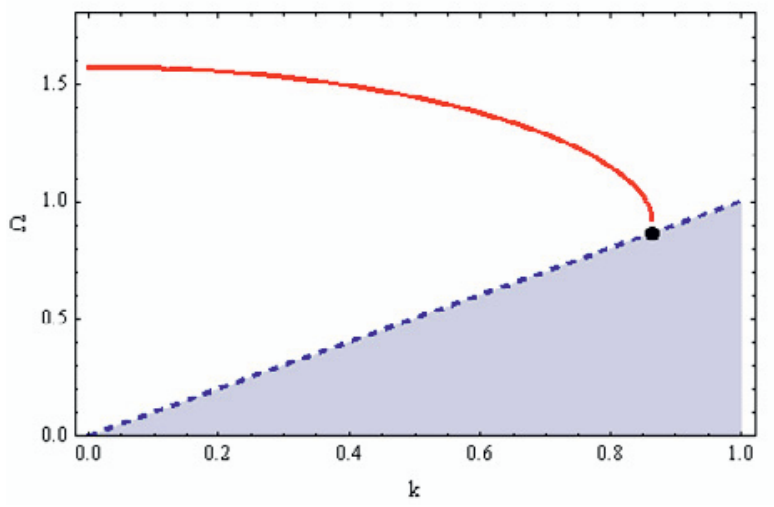

Figure 2. The instability against the spatial inhomogeneity in the stagflation phase. The background dynamical fields, such as Eq.(22), are approximately calculated near the stagflation point. All the parameters are set to be of order one. (solid red line) The instability strength $\Omega$ as a function of $k$. (Blue dashed line) The causal limit line below which the collapse actually takes place. (Black dot) This point corresponds to the fastest mode which alows causal completion of the collapse.

which can be used to the instability analysis. However the result is very complicated to extract any analytic information. Therefore we simply performed numerical check that the instabilities occur in general parameter regions. Fig. 2 is just an example of the calculation.

The Universe is reheated by the particle production mechanism at the stagflation phase immediately after the inflation and the collapse of the condensations into localized structures. Therefore the Universe recovers the Freedman power law evolution. These Bose particles may yield new BEC and mini-stagflation in the later stages of evolution at the total energy naught. Then the same instability will take place to form localized structures again, whose instability region has much larger scale than the original one. In this way, the universe evolves as power law in time on average even though it may repeat intermittent instability/collapse. This is the self-adjustment of BEC and the DE contents in the Universe. We would like to establish this type of dissipative reduction mechanism of the vacuum energy to solve the problem (c) in the introduction although the present analysis is apparently insufficient. Probably we need non-linear instability analysis in our future work.

\section{Summary and discussions}

We have studied the dynamics of inflation based on the BEC cosmology. We tried to be general and minimum as much as possible in this study to construct our model. The unified view of DM and $\mathrm{DE}$ is the starting point of our study, in which we identify them as the normal and condensed part, respectively, of some boson field. Extending this unification in the early Universe, we identified the inflaton field as the condensation of the boson field. Assuming the attractive interaction between the boson fields, we can generally derive the stagflation phase, in which the cosmic expansion temporary ceases while the inflaton field develops.

We have analyzed the instability of the uniform fields during this stagflation phase. This instability triggers the collapse of the fields to form localized structures. Some of the portion of the collapsed boson field may form black holes since the bosons cannot have enough pressure if the total mass 
goes beyond the Kaup mass[5]. These inhomogeneities prevent the Universe to implode, which were inevitable if the Universe remains spatially uniform.

Particle production due to the rapid evolution of the inflaton field reheats the Universe. This particle production will be most effective near the stagflation point where $|\dot{\phi}|^{2}$ becomes large while the uniformity of the field $\phi$ remains. The detailed analysis on the evolution of the vacuum energy goes beyond the present linear analysis although we are planning to perform non-linear calculations in the near future.

As discussed in the last section, the evolution of the Universe may not at all smooth and even but may repeat intermittent instability phases. This possibility should be analyzed more seriously in the above study.

\section{References}

[1] S. Weinberg, Rev. Mod. Phys. 61, 1 (2003).

[2] T. Fukuyama, T. Tatekawa and M. Morikawa, JCAP06 033 (2008).

[3] T. Fukuyama \& M. Morikawa, Phys. Rev. D80, 063520 (2009).

[4] M.Yu.Khlopov, B.A.Malomed, and Ya.B.Zeldovich, Mon. Not. R. astr. Soc. 215 (1985), 575.

[5] D.J. Kaup, Phys. Rev. 172 (1968), 1331.

[6] G. Felder, A. Frolov, L. Kofman, A. Linde, Phys.Rev.D66, 023507 (2002). 